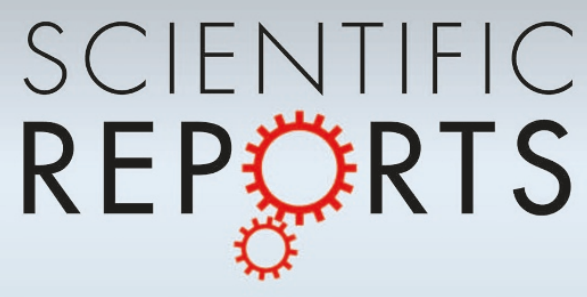

OPEN

SUBJECT AREAS:

NON-SMALL-CELL LUNG

CANCER

MASS SPECTROMETRY

METABOLOMICS

HIGH-THROUGHPUT SCREENING

Received

10 May 2013

Accepted

31 July 2013

Published

23 August 2013

Correspondence and requests for materials should be addressed to H.W.C. (chw8868@ gmail.com)

\section{Direct Characterization of Bulk Samples by Internal Extractive Electrospray lonization Mass Spectrometry}

\author{
Hua Zhang' , Haiwei Gu', Feiyan Yan' , Nannan Wang' ', Yiping Wei' ${ }^{2}$ Jianjun Xu² \& Huanwen Chen'
}

'Jiangxi Key Laboratory for Mass Spectrometry and Instrumentation, East China Institute of Technology, Nanchang, Jiangxi 330013, China, ${ }^{2}$ Department of Cardiothoracic Surgery, The Second Affiliated Hospital of Nanchang University, Nanchang, Jiangxi 330006 , China.

A straight-forward analytical strategy called internal extractive electrospray ionization mass spectrometry (iEESI-MS), which combines solvent extraction of chemicals inside a bulk sample with in situ electrospray ionization mass spectrometry, has been established to directly characterize the interior of a bulk sample with molecular specificity. The method allows both qualitative and quantitative analysis of analytes distributed in a 3-dimensional volume (e.g., $1 \sim 100 \mathrm{~mm}^{3}$ ) of various synthetic and biological matrices (e.g., chewing gum, leaves, fruits, roots, pork, lung tissues) without either mashing the sample or matrix separation. Using different extraction solvents, online chromatographic separation of chemicals inside the sample volume was observed during iEESI-MS analysis. The presented method is featured by the high speed of analysis, high sensitivity, low sample consumption and minimal sample preparation and/or degradation, offering unique possibilities for advanced applications in plant science, clinical diagnosis, catalyst studies, and materials science.

\footnotetext{
( lear visualization of molecules inside bulk samples with high chemical complexity has challenged analytical science for many decades and is of increasing importance in multiple disciplines such as clinical diagnosis, plant science, catalyst studies, materials science, etc. Current analytical techniques usually require multi-step sample pre-treatment (e.g., grinding, extraction, separation, pre-concentration, etc.) to obtain molecular information from the 3-dimensional volume of a bulk sample. Commonly associated with sample pretreatment are biological degradation, chemical reactions, reagent contamination, and material losses. Sensitive and rapid analytical methods are highly demanded that would allow direct analysis of interior chemicals from bulk samples with molecular specificity.

Mass spectrometry (MS) has been increasingly employed for trace analysis in multiple disciplines because of its high sensitivity and molecular specificity ${ }^{1-6}$. Powered by ambient ionization techniques, such as desorption electrospray ionization $(\mathrm{DESI})^{7-9}$, matrix assisted laser desorption/ionization (MALDI) ${ }^{10}$, direct analysis in real time $(\mathrm{DART})^{11}$, low-temperature plasma probe (LTP $)^{12}$, desorption atmospheric pressure chemical ionization $(\mathrm{DAPCI})^{13}$, extractive electrospray ionization $(\mathrm{EESI})^{14-17}$, laser ablation with electrospray ionization $(\mathrm{LAESI})^{18}$ etc., MS has been extended for the direct detection of analytes in complex matrices, with no/minimal sample pretreatment. Numerous applications of ambient mass spectrometry have been documented in the literature, demonstrating that solid surfaces ${ }^{19,20}$, living objects ${ }^{15,21,22}$, complex liquids $^{23,24}$, gases $^{14,25,26}$, and even highly viscous samples $^{23,27}$ can be directly characterized at the molecular level. To date, the majority of efforts have been exerted to the analysis of surfaces, which can be directly sampled by most ambient MS methods ${ }^{7-9}$. Certain applications in chemistry, biology and material science, however, preferably demand simultaneous characterization of chemicals located within the bulk volume rather than on the surfaces of samples. Volumetric molecular information is of particular significance to comprehensively characterize many real-life samples such as plant materials, nanostructured substances, porous catalysts, and mutated tissues.

Herein we report a novel strategy termed as internal extractive electrospray ionization (iEESI) for the straightforward MS analysis of whole-volume (typically $\geq 20 \mathrm{~mm}^{3}$ ) samples (e.g., food, biological tissues) without either mashing/grinding the sample or matrix clean-up. In iEESI, extraction solution (e.g., methanol, water, etc.) charged at a high voltage $( \pm 4.5 \mathrm{kV})$ is directly infused into the 3-dimensional volume of the analyzed sample (e.g., a tissue, leaf, fruit, etc.) through the inserted capillary. The analytes are extracted by the infused solvent and carried along the electric field gradient inside the bulk volume of the sample. Stable electrospray plume is
} 


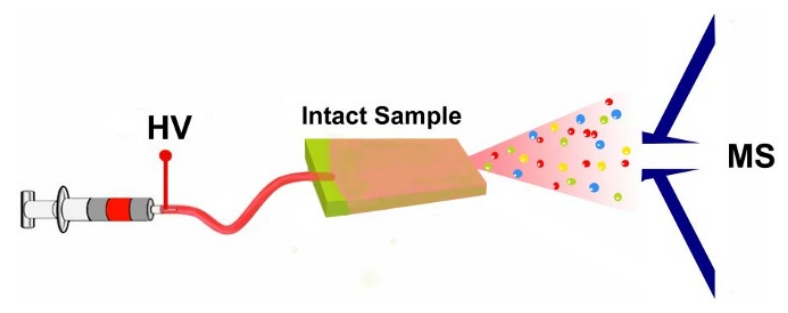

Figure 1 Conceptual illustration of internal extractive electrospray ionization (iEESI).

generated from the opposite sample edge, in front of MS inlet (Fig. 1). Following the iEESI strategy, molecular information of analytes distributed in a bulk volume $\left(1 \sim 100 \mathrm{~mm}^{3}\right)$ of a sample, rather than merely from its surface, can be revealed. The unique mechanism and performance characteristics can render iEESI-MS useful for the direct characterization of bulk samples without affecting their integrity.

\section{Results}

Direct iEESI-MS profiling of analytes inside a bulk volume. In a model experiment, chemical composition of a chewing gum (Kent ${ }^{\circledR}$, USA) was characterized by iEESI-MS (Fig. 2a). Rich MS fingerprint was detected by iEESI-MS in a broad mass range $(\mathrm{m} / z$ 100-1000) with good signal abundance $(3.73 \mathrm{E} 4 \mathrm{cps})$. The chemical ingredients such as glycerol, menthol, menthone, glucose and sucrose (Supplementary Table S1) were successfully identified by iEESI-MS ${ }^{n}$ experiments. The distribution of equally spaced peaks at $\mathrm{m} / z 262$, 348, 434, and 520 (Supplementary Fig. S1) was tentatively attributed to polyvinyl acetate, an essential component of the chewing gum. Remarkably different from iEESI-MS, DESI mass spectrum was dominated by low-mass signals $(m / z<300)$, among which the polyvinyl acetate signals were totally absent (Fig. 2b). In another sampling approach - so-called "leaf spray"28 - a drop of methanol $(5 \mu \mathrm{L})$ was deposited on the gum surface for chemical extraction, and ionic spray was generated by applying high voltage directly to the sample. This approach produced mass spectrum spanning a wide $\mathrm{m} / \mathrm{z}$ range (Fig. $2 \mathrm{c}$ ), but the abundance of most signals was, on the average, 2 orders of magnitude lower than those detected by iEESIMS. Note that the signal density of the "leaf spray" experiment was heavily dependent on the volume of methanol added onto the chewing gum. No signal was detectable if the chewing gum was not sufficiently wet to generate charged plume. Extra experiments were carried out by iEESI-MS to evaluate its feasibility for direct characterization of other bulk samples. Similar to the chewing gum analysis, considerably richer mass spectral patterns for iEESI-MS were also obtained using mammal and plant raw samples including pork meat (Supplementary Fig. S2), garlic leaf (Supplementary Fig. S3), and garlic bulb tissue (Supplementary Fig. S4). Even though default instrument parameters were used in all the three methods considered, care should be taken not to make definitive conclusions based on limited data at this stage.

To explore the sensitivity of iEESI-MS to chemicals on the sample surface, the following reference experiment was conducted. A $0.2 \mu \mathrm{L}$ drop of trinitrotoluene (TNT) aqueous solution (100 ppm) was carefully spiked onto the center of a chlorophytum comosum leaf. The spotted area was cut out of the leaf into a small triangle without contaminating the open cuts. The spiked sample (TNT droplet still wet) was then investigated by iEESI-MS and leaf spray analysis in the negative ion detection mode. Unlike the leaf-spray analysis, no TNT signal was observed by iEESI-MS. Weak isobaric signal at $m / z 227$ recorded by iEESI-MS had different origin, which was confirmed by reference MS/MS analysis of the precursor ions isolated at $m / z 227$ and authentic TNT compound (Supplementary Fig. S5).

To visualize iEESI process, a homemade video was made that shows the real-time color bleaching of canna indica $L$. flower as the anthocyanin pigment is gradually extracted by methanol solvent (Supplementary Video S1). Similar phenomenon was observed (video data not shown) for a strawberry sample. As the pigment in the flesh tissue was dissolved and carried away by the solvent, the color of the strawberry gradually faded. The loss of to pelargonidin3-O-glucoside pigment could be directly revealed in the iEESI mass spectrum of a strawberry, manifested in abundant signal at $m / z 433$ (Supplementary Fig. S6).

Selective extraction of analytes by iEESI-MS. Selective extraction of analytes distributed inside the bulk volume of a raw sample is easily achieved in iEESI by using solvents with different polarity. For example, different analytes were selectively detected from a chewing gum with cyclohexane, water, or methanol as ESI solvent. Dramatically different mass spectra were observed, with regard to both signal composition and total ion intensity (Fig. 3). Generally

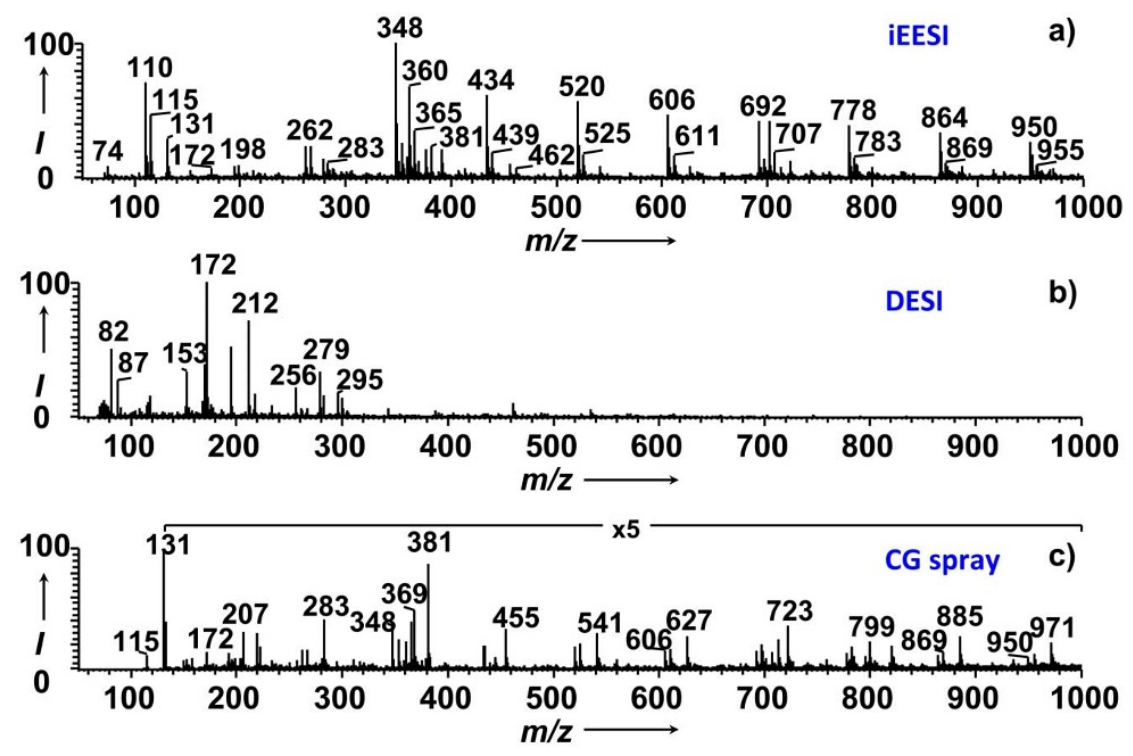

Figure $2 \mid$ Mass spectral patterns recorded from identical chewing gum samples by different ionization techniques. a) iEESI; b) DESI; c) "leaf spray" (direct ESI from the microdroplet spotted on the sample surface). 
low-intensity signals $(\sim 1.23 \mathrm{cps})$ were detected in the mass range of $m / z$ 250-800 (Fig. 3a) from the chewing gum by iEESI-MS with cyclohexane, while considerably richer mass patterns with higher signal abundances $\left(>10^{3} \mathrm{cps}\right.$ ) were obtained using water (Fig. $3 \mathrm{~b}$ ) and methanol (Fig. 3c) under the same experimental conditions. Since cyclohexane is not a very suitable solvent for ESI, the low signal density achieved using cyclohexane might be caused by the low efficiency of either ionization or extraction process of the iEESI. No signal of polar ingredients such as glucose (MW 180) and glycerol (MW 92) was detected by iEESI-MS using cyclohexane. More abundant signals were obtained by iEESI using water and, particularly, methanol as the extraction solvent. Therefore, we attribute the observed difference to the more efficient extraction of these compounds achieved by polar water and methanol solvents as compared to nonpolar cyclohexane. Polar molecules such as glucose $(\mathrm{m} / z 198$ and 221), glycerol $(\mathrm{m} / z 115$ and 131$)$, menthol $(\mathrm{m} / z$ 157), menthone $(\mathrm{m} / z \mathrm{155})$ and sucrose $(\mathrm{m} / \mathrm{z} 360$ and 381$)$ were detected at high abundances in iEESI-MS with water and methanol as the extraction solvent, while the corresponding peaks were absent in the mass spectra using cyclohexane (Fig. 3a). Using water, the dominant mass rage in the mass spectrum was $\mathrm{m} / \mathrm{z} 400-$ 800 (Fig. 3b), while the iEESI-MS fingerprint obtained with methanol spanned a much wider $m / z$ range (100-2000) (Fig. 3c).

Separation effect observed during iEESI process. Interestingly, distinct separation of analytes extracted by the ESI solvent was observed when the distance between the tip of the ESI capillary and the sample edge was increased $(\geq 5 \mathrm{~mm})$. The iEESI-MS spectra of a chewing gum were continuously recorded using methanol as the extraction solvent. The time required to detect analytes originated from the chewing gum varied dramatically from $20 \mathrm{~s}$ to $60 \mathrm{~min}$, depending on the distance of between the ESI tip and the sample edge, the solvent flow rate, air temperature and humidity. With low flow rates $(1 \mu \mathrm{L} / \mathrm{min})$, long distance $(10 \mathrm{~mm})$ and dry ambient air $\left(25^{\circ} \mathrm{C}, 20-25 \%\right.$ R.H. $)$, it took $60 \mathrm{~min}$ to observe a chewing gum spectrum (Fig. 4a). The spectral pattern was unchanged for about $20 \mathrm{~min}$, during which the mass spectra was dominated by low-mass signals (e.g., protonated menthone, $\mathrm{m} / \mathrm{z}$ $155)$, probably because the small polar chemicals were relatively more volatile than the chemicals detected later on. The spectrum recorded after $80 \mathrm{~min}$, on the other hand, revealed abundant signals of large chemicals including polymer molecules (Fig. 4b), suggesting significant chromatographic effects on the time scale of iEESI experiment. With high flow rates $(4 \mu \mathrm{L} / \mathrm{min})$, short distance (4 mm) and humid ambient air $\left(25^{\circ} \mathrm{C}, 65 \%\right.$ R.H. $)$, only $20 \mathrm{~s}$ was required for iEESI to record the first spectrum (Fig. S7a) of a chewing gum sample, which shows the spectral pattern similar to that in Fig. 4a. Under the same conditions, polymer signals (Fig. S7b) were abundantly detected after $4 \mathrm{~min}$ by iEESI-MS from the same chewing gum sample.

To further validate the separation effect, experiments were performed by replacing the chewing gum using radish taproot tissue, which was spiked with $2 \mu \mathrm{L}$ aniline $(2.5 \mathrm{ppm})$ and methylene blue dye $(2.5 \mathrm{ppm})$ methanol/water solution $(1: 1, \mathrm{v} / \mathrm{v})$. The selected ion current of protonated aniline $(\mathrm{m} / z$ 94) was well separated from that of methylene blue cations $(\mathrm{m} / z$ 284) in the time domain (Fig. $4 \mathrm{c})$. Similar separation effect was observed for other compounds such as glucose, choline, and amino acids in the radish tissue (Fig. S8). Note that significant peak tailing was observed in most ion chromatograms detected in iEESI-MS. This was attributed to the imperfect separation conditions for the natural tissue samples. A reference experiment, which was conducted using a fused silicon capillary (detailed in the Supplementary Information, Figure S9) filled with C18 stationary phase (bead size $5 \mu \mathrm{m}$, Agilent, USA), showed full separation of aniline (retention time $\sim 2.33 \mathrm{~min}$ ) and arginine (retention time $\sim 4.15 \mathrm{~min}$ ) with well-shaped chromatographic peaks with full width at half maximum (FWHM) about $20 \mathrm{~s}$.

Quantitation by iEESI-MS. The potential of iEESI for quantitative analysis was experimentally investigated. Bulk samples of pork meat $(3 \times 2 \times 2 \mathrm{~cm})$ spiked with different amounts of salbutamol were analyzed by iEESI-MS/MS. Salbutamol was identified based on the observation of characteristic fragment ions $(\mathrm{m} / z 222$, Supplementary Fig. S10) in MS/MS mode. A linear correlation was found between the signal intensity for $m / z 222$ in iEESI-MS/MS and the concentration of salbutamol in pork meat $(\mathrm{y}=0.6971 \mathrm{x}+5.79$, Fig. 5a). The limit of detection (LOD, $\mathrm{S} / \mathrm{N}=5$ ) of this method was estimated as $0.0399 \mu \mathrm{g} / \mathrm{L}$ for salbutamol, and relative standard deviation (RSD) was in the range of $6-15 \%$ (7 pork samples). Note that for better results, the ESI capillary tip should be carefully positioned with the same distance (e.g., $2 \mathrm{~mm}$ ) away from the sample edges. Otherwise, the signal intensities for 7 samples spiked

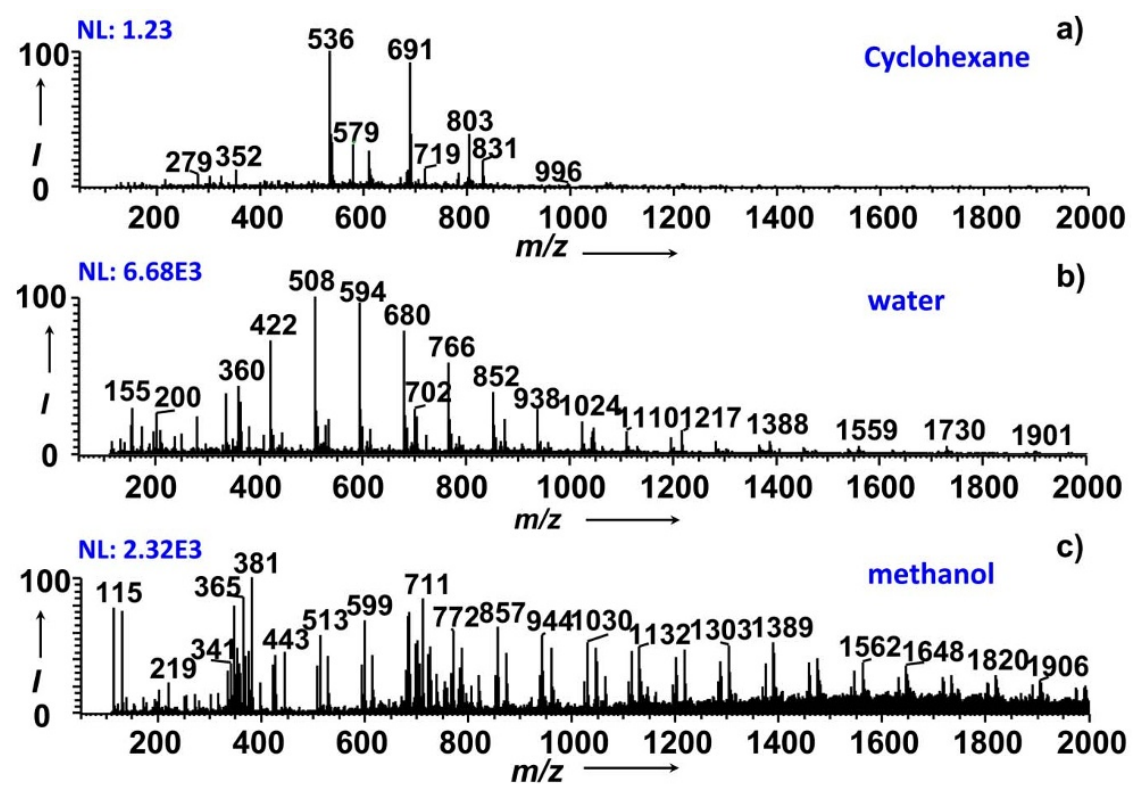

Figure 3 Selective extraction of analytes distributed inside the bulk volume of a chewing gum using different solvents for iEESI-MS analysis. a) cyclohexane; b) water; c) methanol. 


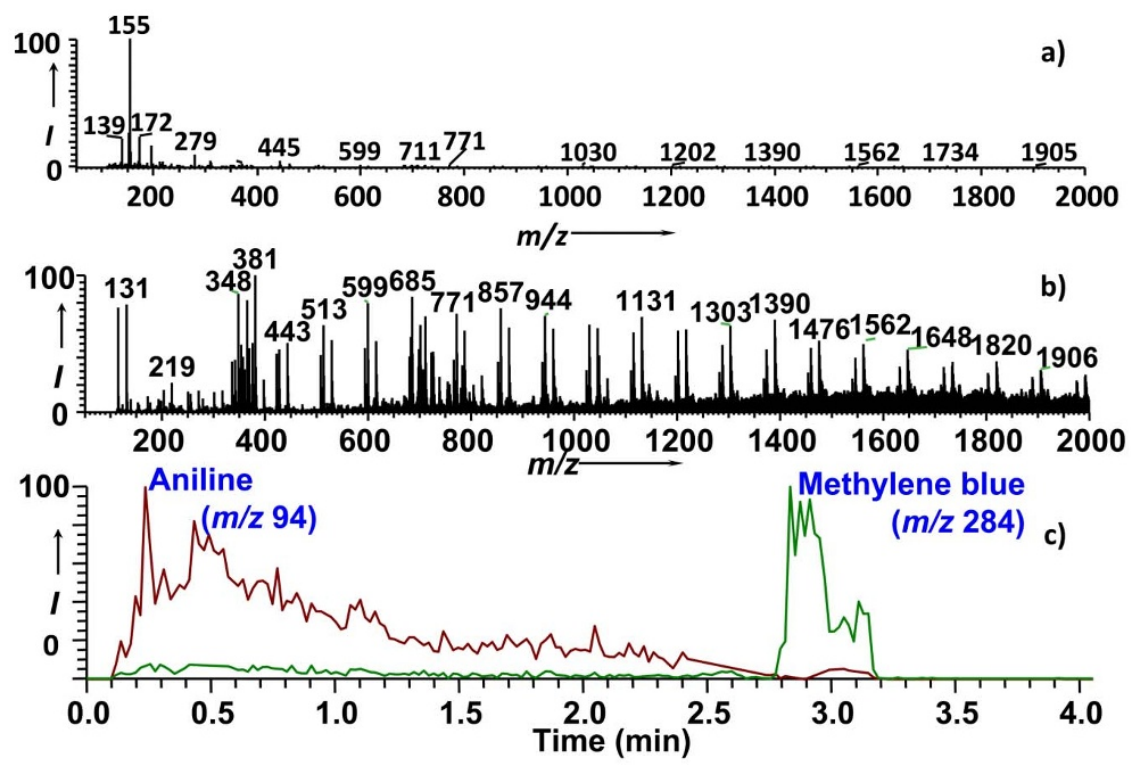

Figure 4 | Separation effects observed in iEESI-MS. a) a typical mass spectrum recorded after 60 min from the beginning of the experiment; b) a typical mass spectrum recorded after $80 \mathrm{~min}$; c) selected chromatographic traces of aniline (m/z 94) and methylene blue dye (m/z 284) solution spiked into a fresh radish taproot sample.

with same concentration could vary substantially, because the sample volume which was actually analyzed for each individual piece were quite different. Although with lower signal intensities, linear signal responses were also obtained using pork samples of smaller sizes, showing that the minimal sample required for this experiment was less than $1 \mathrm{~mm}^{3}$.

Lung cancer tissues sample analysis. The features of iEESI-MS make it potentially attractive for rapid in situ identification of cancer tissue during surgical operations. In a preliminary experiment, full scan iEESI mass spectra were recorded from a set (20 samples in total) of 10 small pieces of clinically cut lung cancer tissue samples (Supplementary Fig. S11a) and 10 small pieces of the pericarcinomatous tissue samples (Figure S11b). As a result, the mass spectral pattern of each lung cancer tissue sample was distinguished from the corresponding pericarcinomatous ones. The difference between these samples on the molecular level was detected by iEESI-MS analysis within 2 min which included the time required for sample loading. In the principal component analysis (PCA) score plot (Fig. 5b), the lung cancer samples were clearly separated from the normal lung tissue.

\section{Discussion}

Our results demonstrate that iEESI enables direct chemical characterization of the interior in an intact sample without destroying the sample integrity. The analytical performance of iEESI is notably different from that of DESI and previously reported "leaf spray" ionization ${ }^{28}$, especially in terms of sensitivity and the number of detected compounds. The higher chemical capacity of the method was observed for the chewing gum sample (Figure 2), as well as for mammal and plant raw samples including pork meat (Supplementary Fig. S2), garlic leaf (Supplementary Fig. S3) and intact garlic bulb (Supplementary Fig. S4). The observed spectral enhancement in iEESI-MS suggests considerable contribution of analytes extracted from the bulk sample volume in addition to those extracted from the surface.

Most of ambient MS methods rely on surface desorption. Volumetric information provided by those methods is limited to the penetration depth of the employed desorption source and range from the $\mu \mathrm{m}$ scale (e.g., laser or plasma desorption) to $\mathrm{mm}$ (e.g., solvent extraction, such as in DESI or leaf spray). The major contribution to generated mass spectra is still derived from surface chemicals. The introduced iEESI-MS approach reveals essentially orthogonal behavior. The desorption/extraction is performed from inside the sample in iEESI. At given concentrations (e.g., $1 \mathrm{ng} / \mathrm{mm}^{2}$ for surface analysis), the total amount of the analyte in a 3-D volume (e.g., $20 \mathrm{~mm}^{3}$ ) is much higher than that on a surface of $10 \mathrm{~mm}^{2}$, a typical sampling area for surface analysis using ambient desorption ionization techniques. Therefore, the iEESI method is even more sensitive for trace
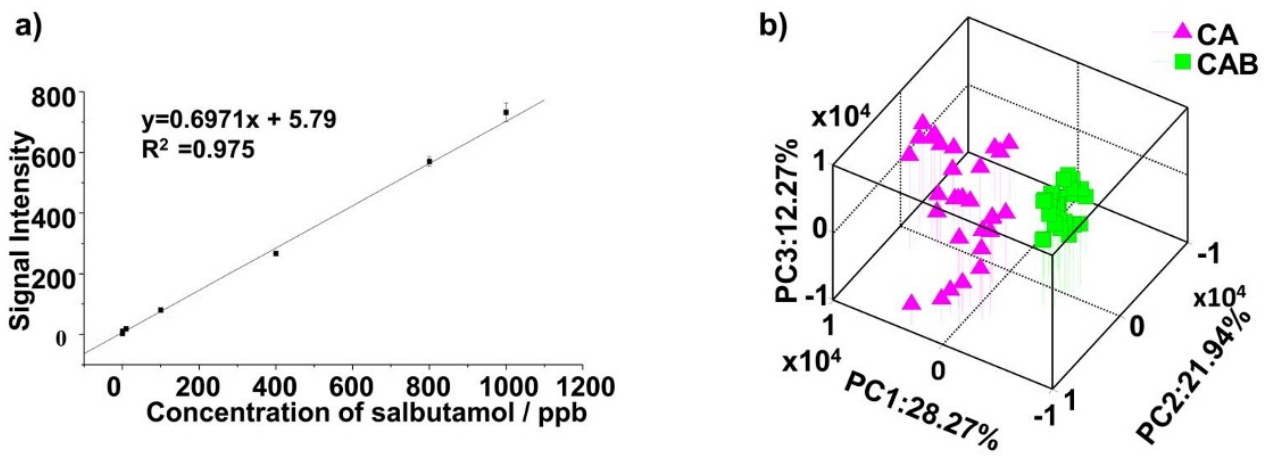

Figure 5 Rapid analysis of mammal tissue samples by iEESI-MS. a)The calibration curve of salbutamol in pork meat samples; b) the 3D PCA score plot of iEESI-MS data collected from 10 sets of lung cancer (CA) and pericarcinomatous (CAB) tissue samples. 
analysis. Furthermore, iEESI detects the interior chemicals rather than those on the surface. This performance feature of iEESI was demonstrated using the analysis of TNT compound spiked on the leaf surface. While easily visible by surface-specific ambient methods such as "leaf spray", no TNT signal was detected by iEESI-MS (Supplementary Fig. S5). Because the chemical composition of sample surfaces and interior are often very different, the capability of iEESI to discriminate against surface compounds can be important for sensitive and selective characterization of inner chemicals.

Chromatographic capacity is another key feature of iEESI-MS (Figures 3 and 4). In most ambient methods, analytes desorbed from sample surfaces are directly sampled for MS detection without any pre-separation in space and/or time. As a result, ambient analyses commonly suffer from the extreme sample complexity of real-life samples, requiring more advanced MS instruments to provide sufficient resolving power and specificity of detection. In iEESI, the sample volume serves a natural chromatographic medium for extracted species. Notable separation effects are observed for the analyzed volumes of $\geq 20 \mathrm{~mm}^{3}$. As demonstrated, the fingerprint complexity can be tailored by the selection of ESI solvent (Figure 3). In addition to the $\mathrm{m} / z$ signals in MS domain, analytes in iEESI-MS can be characterized by the time of elution and affinity to particular extraction solvent, which can be used to enhance the chemical specificity of MS identification, similar to LC-MS or GCMS. This feature is of significance for profiling metabolites of differential chemiphysical properties from tissue samples using commonly available MS instruments, allowing sequential detection of metabolites grouped by similar chromatographic behaviors with specifically-tailored experimental conditions (e.g., voltages, solvent, etc.).

The high affinity of iEESI-MS to interior chemicals can be of particular interest for the recognition of deliberate chemical contamination in many fields such as food industry. Ambient ionization techniques including DESI, DART, and DAPCI are often used for the detection of hazardous agents, such as adulterants, drugs or pesticides on food surfaces. However, the abundance of those compounds on the sample surface can be substantially lower than in the bulk volume. For example, pesticides can be efficiently removed from fruit surface post-harvest by tedious washing or chemical treatment. In this case, pesticide treatment can remain undiscovered by surface methods, although pesticides can still be abundantly present inside the fruit volume. Our experiments demonstrate that iEESI-MS can be a practical method to reveal volumetric chemical contaminants. Targeted quantitative detection of salbutamol in pork meat samples was demonstrated with high accuracy and sensitivity as well as low sample consumption (Fig. 5a).

Finally, our data suggest the potential of iEESI-MS in clinical diagnosis. Different metabolites are commonly non-evenly distributed across mammalian tissues. Therefore, whole-volume analysis can be necessary for the unbiased characterization of a tissue sample. The demonstrated possibility to discriminate lung cancer tumors with iEESI-MS (Fig. 5b) encourages further experiments to validate the method using large sample sets, which are currently underway in our laboratory.

To conclude, a novel analytical strategy has been established for the selective extraction of analytes distributed in the interior volume of a bulk sample, rather than on its surface, for direct electrospray ionization and simultaneous MS detection. Distinct chromatographic separation and solvent-specific release of analytes were observed during the internal extractive electrospray ionization process. The possibility of targeted quantitative detection and biological tissue differentiation were demonstrated. The method does not require any sample pretreatment and is featured by high analysis speed, high sensitivity and low sample consumption. The experimental data reported here suggest high potential of iEESI-MS in multiple disciplines such as plant science, clinic diagnosis, catalyst studies, materials science, etc.

\section{Methods}

Sample collection. The chemical reagents such as methanol (ROE Scientific ${ }^{\circledR}$ Inc. Newark, USA), TNT (Chem Service ${ }^{\circledR}$, Inc. PA, USA), salbutamol (Sinopharm Chemical Reagent Co. Ltd (Shanghai)) were used with the highest purity grade available. Strawberries, garlic leaves, garlic bulbs, radish taproots, pork meat and chewing gum were purchased from a local supermarket. The Chlorophytum comosum $L$. leaves and the Canna indica $L$. flowers were collected in the park area of ECIT campus. The lung cancer tissue and pericarcinomatous tissue samples were clinically obtained from the Second Affiliated Hospital of Nanchang University, with full consent from the volunteers. Special care was taken to ensure safety of the operators when performing lung cancer tissue measurements, with onsite guidance from doctors of the hospital.

Mass spectrometry analysis. All the experiments were carried out using an LTQ-MS instrument (Thermal Fisher, San Jose, CA) installed with a homemade iEESI, DESI and "leaf spray" source. Briefly, in iEESI experiments solvent (e.g., methanol, water, etc.) was directly infused at flow rates of 1-8 $\mu \mathrm{L} / \mathrm{min}$ into the bulk volume of a sample (e.g., a tissue, leaf, etc.) through the inserted fused silicon capillary (ID $0.25 \mathrm{~mm}$, OD $0.30 \mathrm{~mm}$, Agilent, USA) at a high voltage of $\pm 4.5 \mathrm{kV}$. The capillary end was inserted into the bulk volume of an intact sample piece, allowing ca. $2 \sim 5 \mathrm{~mm}$ distance between the capillary tip and the sample edge. The analytes distributed within a bulk volume of the sample were extracted by the charged solvent and carried along the electric field inside the sample. Stable electrospray plume was generated from the opposite sample edge (Supplementary Information, Video S1), in front of the MS inlet. The iEESI setup was aligned coaxially with the ion entrance of MS instrument, such that the analyte ions were directly transferred for mass analysis. The samples, such as leaf and animal tissues, were directly analyzed without matrix clean up or reagent deposition. The optimized temperature of the heated capillary was set to be $150^{\circ} \mathrm{C}$. Other parameters were set as default values of the instrument, and no further optimization was performed. The reference experiments of spray-MS and DESI-MS were performed in conventional way according to the descriptions given in ref ${ }^{8,28}$. Experimental details can be found in the Supplementary Information.

Statement. The present research was adhered to the tenets of the Declaration of Helsinki ${ }^{29,30}$, and approved by the Ethics Committee of the East China Institute of Technology and the Hospital Institutional Review Board of the Second Affiliated Hospital to Nanchang University. Additionally, the benefits and risks of this study were clearly presented to the volunteers, and thereafter the written consent was obtained if they agreed to join the study.

1. Elias, J. E. \& Gygi, S. P. Target-decoy search strategy for increased confidence in large-scale protein identifications by mass spectrometry. Nat. Methods 4, 207-214 (2007).

2. Domon, B. \& Aebersold, R. Review - Mass spectrometry and protein analysis. Science 312, 212-217 (2006).

3. Cooks, R. G., Ouyang, Z., Takats, Z. \& Wiseman, J. M. Ambient mass spectrometry. Science 311, 1566-1570 (2006).

4. Heck, A. J. R. Native mass spectrometry: a bridge between interactomics and structural biology. Nat. Methods 5, 927-933 (2008).

5. Cravatt, B. F., Simon, G. M. \& Yates, J. R. The biological impact of massspectrometry-based proteomics. Nature 450, 991-1000 (2007).

6. Olsen, J. V. et al. Global, in vivo, and site-specific phosphorylation dynamics in signaling networks. Cell 127, 635-648 (2006).

7. Eberlin, L. S., Ifa, D. R., Wu, C. P. \& Cooks, R. G. Three-Dimensional Vizualization of Mouse Brain by Lipid Analysis Using Ambient Ionization Mass Spectrometry. Angew. Chem. Int. Edit. 49, 873-876 (2010).

8. Takats, Z., Wiseman, J. M., Gologan, B. \& Cooks, R. G. Mass spectrometry sampling under ambient conditions with desorption electrospray ionization. Science 306, 471-473 (2004).

9. Wiseman, J. M., Ifa, D. R., Song, Q. Y. \& Cooks, R. G. Tissue imaging at atmospheric pressure using desorption electrospray ionization (DESI) mass spectrometry. Angew. Chem. Int. Edit. 45, 7188-7192 (2006).

10. Gopal, J., Hasan, N., Manikandan, M. \& Wu, H. F. Bacterial toxicity/compatibility of platinum nanospheres, nanocuboids and nanoflowers. Sci. Rep. 3, 1260; DOI:10.1038/srep01260 (2013).

11. Yew, J. Y., Cody, R. B. \& Kravitz, E. A. Cuticular hydrocarbon analysis of an awake behaving fly using direct analysis in real-time time-of-flight mass spectrometry. $P$. Natl. Acad. Sci. Usa. 105, 7135-7140 (2008).

12. Liu, Y. Y. et al. Imaging Mass Spectrometry with a Low-Temperature Plasma Probe for the Analysis of Works of Art. Angew. Chem. Int. Edit. 49, 4435-4437 (2010).

13. Yang, S. P. et al. Detection of Melamine in Milk Products by Surface Desorption Atmospheric Pressure Chemical Ionization Mass Spectrometry. Anal. Chem. 81, 2426-2436 (2009).

14. Chen, H. W., Wortmann, A., Zhang, W. H. \& Zenobi, R. Rapid in vivo fingerprinting of nonvolatile compounds in breath by extractive electrospray ionization quadrupole time-of-flight mass spectrometry. Angew. Chem. Int. Edit. 46, 580-583 (2007).

15. Chen, H. W., Yang, S. P., Wortmann, A. \& Zenobi, R. Neutral desorption sampling of living objects for rapid analysis by extractive electrospray ionization mass spectrometry. Angew. Chem. Int. Edit. 46, 7591-7594 (2007). 
16. Chen, H. W. et al. Sensitive Detection of Native Proteins Using Extractive Electrospray Ionization Mass Spectrometry. Angew. Chem. Int. Edit. 49, 3053-3056 (2010).

17. Li, M. et al. Facilitated Diffusion of Acetonitrile Revealed by Quantitative Breath Analysis Using Extractive Electrospray Ionization Mass Spectrometry. Sci. Rep. 3, 1205; DOI:10.1038/srep01205 (2013).

18. Nemes, P. \& Vertes, A. Laser ablation electrospray ionization for atmospheric pressure, in vivo, and imaging mass spectrometry. Anal. Chem. 79, 8098-8106 (2007).

19. Qian, K. et al. Laser Engineered Graphene Paper for Mass Spectrometry Imaging. Sci. Rep. 3, 1415; DOI:10.1038/srep01415 (2013).

20. McDonnell, L. A. \& Heeren, R. M. A. Imaging mass spectrometry. Mass. Spectrom. Rev. 26, 606-643 (2007)

21. Wollscheid, B. et al. Mass-spectrometric identification and relative quantification of N-linked cell surface glycoproteins. Nat. Biotechnol. 27, 378-386 (2009).

22. Louie, K. B. et al. Mass spectrometry imaging for in situ kinetic histochemistry. Sci. Rep. 3, 1656-1656 (2013).

23. Law, W. S. et al. Rapid Characterization of Complex Viscous Liquids at the Molecular Level. Angew. Chem. Int. Edit. 48, 8277-8280 (2009).

24. Dettmer, K., Aronov, P. A. \& Hammock, B. D. Mass spectrometry-based metabolomics. Mass. Spectrom. Rev. 26, 51-78 (2007).

25. Gamez, G. et al. Real-time, in vivo monitoring and pharmacokinetics of valproic acid via a novel biomarker in exhaled breath. Chem. Commun. 47, 4884-4886 (2011).

26. Peng, G. et al. Diagnosing lung cancer in exhaled breath using gold nanoparticles. Nat. Nanotechnol. 4, 669-673 (2009).

27. Li, X., Hu, B., Ding, J. H. \& Chen, H. W. Rapid characterization of complex viscous samples at molecular levels by neutral desorption extractive electrospray ionization mass spectrometry. Nat. Protoc. 6, 1010-1025 (2011)

28. Liu, J. J., Wang, H., Cooks, R. G. \& Ouyang, Z. Leaf Spray: Direct Chemical Analysis of Plant Material and Living Plants by Mass Spectrometry. Anal. Chem. 83, 7608-7613 (2011).
29. Goodyear, M. D. E., Krleza-Jeric, K. \& Lernmens, T. The Declaration of Helsinki. Brit. Med. J. 335, 624-625 (2007).

30. Zion, D., Gillam, L. \& Loff, B. The Declaration of Helsinki, CIOMS and the ethics of research on vulnerable populations. Nat. Med. 6, 615-617 (2000).

\section{Acknowledgments}

This work was supported by National Natural Science Foundation of China (NSFC) (No. 21225522, No. 21175019).

\section{Author contributions}

H.C. and H.G. conceived and designed the research; H.Z. and H.C. analysed the experimental data and wrote the manuscript. H.C. contributed significantly to the discussion of results and manuscript refinement. Y.W. and J.X. performed cancer tissue sample preparation and clinical consultation. H.Z., F.Y. and N.W. performed the experiments.

\section{Additional information}

Supplementary information accompanies this paper at http://www.nature.com/ scientificreports

Competing financial interests: The authors declare no competing financial interests.

How to cite this article: Zhang, H. et al. Direct Characterization of Bulk Samples by Internal Extractive Electrospray Ionization Mass Spectrometry. Sci. Rep. 3, 2495; DOI:10.1038/ srep02495 (2013).

(c) (i) (-) This work is licensed under a Creative Commons Attribution-

BY NC No NonCommercial-NoDerivs 3.0 Unported license. To view a copy of this license, visit http://creativecommons.org/licenses/by-nc-nd/3.0 